\title{
Place et rôle des jeux numériques dans le développement des activités narratives dans une classe de FLE
}

\section{Video games and narrative activities in a French as a foreign language classroom}

\author{
Krzysztof Kotuła \\ Université Marie Curie-Skłodowska de Lublin \\ christophe.kotula@gmail.com
}

\begin{abstract}
The aim of the present article is to focus on the potential of video games as a tool enabling the language teacher to transform the learning experience in the FL classroom. Apart from presenting various theoretical aspects of integrating computer-enhanced ludic techniques into language teaching, learner discourse excerpts will be analysed in order to determine the extent to which video games implementation can enhance classroom interaction. Our main aim will be to list various contexts in which narrative activities can manifest themselves during French as a foreign language lessons. As we will see, learners can be encouraged to create stories not only in reaction to cinematic sequences but also when attempting to reconstruct events which occurred earlier in the game or while recalling events from an earlier gaming session.
\end{abstract}

Keywords: French as a foreign language, narration, CALL, video games

\section{INTRODUCTION - PLACE DU JEU VIDÉO DANS L’ENSEIGNEMENT DES LANGUES}

L'utilisation du jeu vidéo en classe de langue est un sujet qui jouit d'une popularité croissante parmi les spécialistes dans le domaine de l'apprentissage des langues assisté par ordinateur. Depuis quelques années, le nombre d'études qui y sont consacrées augmente exponentiellement : ceci se traduit non seulement par le nombre croissant d'articles apparaissant ponctuellement dans les prestigieuses 
revues telles que ReCALL, Language Learning \& Technology, Computer Assisted Language Learning ou CALICO Journal, mais également par l'apparition d'initiatives d'ampleur plus importante, telle que la publication de recueils d'articles ${ }^{1}$ ou de numéros spéciaux de revues ${ }^{2}$ consacrés exclusivement à ce sujet. Une grande majorité des recherches consacrées à l'enseignement de langues «gamifié » dans le milieu institutionnel - thématique qui nous intéresse $\mathrm{ici}^{3}$ - se concentrent avant tout sur deux questions distinctes : d'une part, sur la manière optimale d'adapter le jeu à la réalité de la classe de langue et le choix du type de jeu dont les caractéristiques seraient les plus appropriées au contexte éducatif; d'autre part, sur les compétences langagières et générales de l'apprenant qu'il est possible de développer grâce à des activités vidéoludiques. Ces deux problèmes s'avèrent souvent délicats, car un jeu vidéo est un logiciel qui est - en principe - créé dans le but de divertir et non pas d'instruire $^{4}$; ainsi, son utilisation efficace dans l'apprentissage peut-elle se révéler particulièrement difficile.

Or, de nombreux chercheurs s'accordent à dire que l'effort consacré à la tâche d'intégrer les activités ludiques dans le processus d'apprentissage peut s'avérer particulièrement fructueux. Les jeux ont le potentiel de transformer l'expérience de l'apprentissage et de stimuler le développement de nombreuses capacités des élèves, parmi lesquelles Mawer et Stanley énumèrent « creative problem solving, calculated risk taking, persistence, attention to detail [and] effective collaboration » (Mawer \& Stanley, 2011, p. 10). De nombreux avantages se présentent également dans le contexte plus restreint, celui de l'apprentissage des langues, et plus particulièrement du développement de l'expression orale ; ainsi, comme le souligne Thomas,

[...] games can be motivating, reduce anxiety, focus learners on communicating in the target language rather than on using correct linguistic structures, provide more introverted students with a greater range of opportunities for self-expression and create an informal atmosphere that enhances learner receptiveness (Thomas, 2012, p. 11).

\footnotetext{
${ }^{1}$ Tels que Digital Games in Language Learning and Teaching publié en 2012 par Palgrave Macmillan sous la direction de Hayo Reinders.

${ }^{2}$ Par exemple, Game-informed L2 Teaching and Learning, numéro spécial de Language Learning \& Technology paru en juin 2014 (vol. 18, no. 2).

${ }^{3}$ Il existe également des recherches se concentrant sur les activités de l'apprenant se situant en dehors de l'école (extramural activities) - telles que la participation à des jeux en ligne massivement multijoueur - et leur impact sur le développement de ses compétences langagières.

${ }^{4}$ La question de la relation entre jeu et apprentissage étant extrêmement complexe, nous allons nous satisfaire de suggérer à tout lecteur intéressé deux lectures essentielles sur le sujet: Don't Bother Me Mom - I'm Learning! de Mark Prensky et What Video Games Have to Teach Us About Learning and Literacy de James Paul Gee. Pour une discussion plus approfondie réunissant les connaissances les plus actuelles sur la question, nous renvoyons au numéro spécial de Educational Psychologist intitulé Psychological Perspectives on Digital Games and Learning, publié en 2015 (vol. 50, no. 4).
} 
L'accent est donc mis avant tout sur l'augmentation du niveau de motivation, la diminution du niveau d'anxiété langagière, ainsi que sur la création d'un cadre propice aux échanges oraux entre les apprenants permettant à ceux-ci de se focaliser avant tout sur le contenu de leur production plutôt que sur sa forme. Se dessine donc l'image de l'interaction libérée des contraintes qui pèsent sur elle et qui rendent souvent la communication dans une classe de langue éloignée des modèles du monde réel ${ }^{5}$.

Il est évidemment justifié de se poser la question d'où découlent ces avantages et quelles sont les caractéristiques propres aux jeux numériques qui décident de leur utilité dans un cours de langue.

Sans doute, l'une des particularités fondamentales des mondes numériques est leur autonomie : dans la réalité virtuelle, chacune des actions des apprenants est suivie d'une réaction appropriée du logiciel. Cela donne aux élèves le sentiment de pouvoir réellement influencer le cours des événements. Ils ne sont pas des spectateurs passifs, mais participent activement dans le développement de l'histoire, et leurs décisions ont un impact réel sur son dénouement. Lorsque le jeu est intégré dans la réalité d'une classe de langue, les apprenants communiquent oralement pour se donner des conseils, ils tentent de deviner quels seront les résultats de leurs actions et ils spéculent sur les conséquences que telle ou telle décision pourra avoir sur la manière dont se déroulera l'intrigue.

Un autre aspect essentiel de l'expérience est lié à la motivation. Les mécanismes intrinsèques du jeu sont construits de façon à encourager constamment le joueur à découvrir les étapes successives du jeu, en grande partie grâce à un système équilibré de récompenses. Comme l'a constaté S. Johnson, « in the gameworld, reward is everywhere. The universe is literally teeming with objects that deliver very clearly articulated rewards [...]. Most of the crucial work in game interface design revolves around keeping players notified of potential rewards available to them » (Johnson, 2005, p. 35). Ainsi, la curiosité du joueur est savamment entretenue, à condition, bien sûr, que le jeu soit bien construit.

Inutile de dire que la mise en œuvre des stratégies vidéoludiques entraîne une profonde mutation du rôle de l'enseignant qui, dans la plupart des cas, peut se libérer de la contrainte de devoir contrôler l'ensemble des processus communicatifs dans la classe. Ainsi, il peut se contenter d'intervenir seulement lorsqu'il le jugera nécessaire pour que le flux d'informations soit maintenu. Les jeux offrent donc, du moins en théorie, une réelle possibilité de transformer l'expérience éducative.

\footnotetext{
${ }^{5}$ Le problème de la spécificité ou de l'« authenticité » (ce deuxième terme devant être utilisé avec grande précaution, voir Piotrowski, 2011, pp. 65-77) de la communication orale dans une classe de langue est très délicat. Nous avons analysé en détail les questions de la spécificité des échanges oraux dans les environnements numériques dans nos deux articles précédents (Kotuła, 2015, 2016).
} 


\section{IMPLÉMENTATION DES TECHNIQUES VIDÉOLUDIQUES - DE LA THÉORIE À LA PRATIQUE}

Bien évidemment, pour que tous les avantages potentiels mentionnés ci-dessus puissent se matérialiser, plusieurs conditions doivent être remplies. Un enseignant désirant intégrer les solutions vidéoludiques dans le processus d'enseignement de langue doit faire face à un large éventail de problèmes en commençant par le choix $\mathrm{du}$ logiciel approprié, en passant par les aspects purement techniques (p. ex. choix d'interface), et en terminant par les questions relatives à l'organisation de l'apprentissage (p. ex. attribution des rôles respectifs aux participants).

Le premier problème est évidemment fondamental. Nous ne mettons pas en question la capacité des jeux numériques à engager les émotions et à stimuler l'imagination des utilisateurs ; il serait pourtant trop hâtif d'en déduire que leur intégration dans le curriculum est toujours souhaitable. Certains types de jeux, notamment les jeux d'action, faisant essentiellement appel à l'habileté et aux réflexes d'un joueur individuel, ne s'adapteront en principe pas bien à la réalité d'une classe de langue.

Ainsi, dans nos recherches, nous nous sommes principalement tourné vers le jeu d'aventure, défini comme « jeu dont l'intérêt prédominant se focalise sur la narration plutôt que sur les réflexes et l'action $»^{6}$. À nos yeux, ses éléments constitutifs essentiels, c'est-à-dire l'accent mis sur la découverte d'une histoire, sur l'exploration $\mathrm{du}$ monde et sur l'interaction avec des personnages en font un outil privilégié permettant de stimuler l'interaction entre les apprenants. Dans les jeux d'aventure, le participant incarne un personnage qui évolue dans un environnement virtuel qui ressemble souvent au monde réel, même lorsque ce dernier en présente une version plus ou moins altérée (monde post-apocalyptique, monde dans lequel sont présents des éléments surnaturels etc.). L'objectif principal du protagoniste est de l'explorer et d'essayer d'en percer les secrets en découvrant l'histoire qui se dévoile successivement à ses yeux.

Quant aux questions techniques, comme notre priorité était de constituer une communauté de collaborateurs communiquant dans le but de résoudre les problèmes qui surgissent lors du jeu, nous avons voulu créer un environnement qui favoriserait les échanges oraux entre les apprenants. Ainsi, nous avons privilégié le tableau interactif qui, d'une part, grâce à une surface d'affichage de dimensions importantes, permet à l'ensemble des participants de suivre aisément le déroulement des événements, et, d'autre part, rend possible la manipulation des objets virtuels avec le toucher. Grâce à ce type d'interface, les gestes des utilisateurs sont immédiatement traduits en actions concrètes. Il est donc tout à fait envisageable qu'un élève formule une instruction susceptible de causer une réaction appropriée du destinataire (telle que « appuie sur le bouton de l'ascenseur ») et que l'autre s'exécute en touchant la

\footnotetext{
${ }^{6}$ https://fr.wikipedia.org/wiki/Jeu_d\%27aventure
} 
surface du tableau et en actionnant ainsi, de la manière la plus intuitive possible, la fonction correspondante du logiciel. L'interface tactile renforce l'impression de contact direct avec la réalité créée par l'ordinateur.

Finalement, quant à l'organisation des échanges, l'interaction se déroule le plus souvent selon le modèle suivant : à un des élèves, choisi au hasard, est confiée la tâche de manipuler le TBI, alors que les autres lui donnent des instructions ou conseils. En même temps, l'ensemble des joueurs constitue une communauté de réflexion qui échange des idées sur la manière optimale de résoudre les problèmes qui émergent au cours du jeu. Il est alors logique de supposer que les séquences qui apparaîtront le plus souvent vont être celles où les apprenants s'échangeront des conseils et instructions, spéculeront sur les actions à entreprendre afin d'atteindre l'objectif du jeu, s'interrogeront sur les erreurs commises au cours du jeu et émettront des hypothèses sur les moyens d'éviter la défaite suivante.

\section{PLACE DE LA NARRATION DANS LES JEUX VIDÉO}

La description des activités vidéoludiques que nous avons donnée dans la section précédente risque de créer une impression que l'interaction entre les apprenants qui y participent se limite principalement à de simples échanges de phrases formulées à l'impératif du type «va à $\mathrm{x}$ », 《 prends $\mathrm{y}$ », 《 ouvre $\mathrm{z}$ » etc. suivies d'une réaction appropriée de l'élève responsable de manipuler le TBI. Notre lecteur pourrait alors se demander si la narration - phénomène autour duquel s'organisent les études réunies dans le présent numéro - peut se manifester dans ce contexte particulier. Comme nous le verrons dans la suite de l'article, plusieurs occurrences de la narration ont pu être relevées dans le corpus des leçons enregistrées ${ }^{7}$. Notre objectif est de recenser les divers contextes dans lesquels la narration prend une place prépondérante.

Pour les besoins de notre étude, nous allons reprendre la définition de la narration telle qu'elle est formulée dans les travaux de U. Paprocka-Piotrowska. Ainsi, un texte narratif proprement dit est composé d'une série de macro-séquences (appelées macro-propositions). Ces éléments constitutifs, au nombre de cinq, apportent chacun une réponse à des questions (implicites) qui permettent de comprendre le déroulement de l'histoire : qui ? quoi ? quand ? où ? (orientation), et alors il est arrivé que ? (complication), et après, qu'est-ce qu'il est arrivé ? (développement), cela a mené à ? (résolution) et comment cela s'est-il terminé ? (conclusion). Les trois macropropositions centrales (complication, développement et résolution) sont considérées

\footnotetext{
${ }^{7}$ Toutes les leçons dont les fragments que nous allons présenter ici ont été enregistrées pendant les cours de français dirigés par l'auteur du présent article au Lycée d'enseignement général no. 5 à Cracovie au cours des années 2014-2017. Le niveau de langue des apprenants correspondait, selon le groupe, au niveau A2/B1-B2 du CECR.
} 
comme essentielles, car elles forment la charpente du récit, tandis que les deux restantes sont facultatives (Paprocka-Piotrowska, 2013, p. 22).

Bien évidemment, la nature même du récit impose une certaine organisation interne de l'intrigue. Comme le souligne U. Paprocka-Piotrowska, « les faits doivent être présentés dans un ordre qui devrait permettre à son interlocuteur de comprendre la suite événementielle et sa dimension causale " (Paprocka-Piotrowska, 2013, p. 64). Nous nous attendons donc en général à ce que les événements racontés s'inscrivent dans une suite chronologique et se développent selon une logique qui implique l'existence d'une relation de cause à effet entre eux. La dimension temporelle est également essentielle. En analysant les discours narratifs des apprenants débutants du FLE, M. Buysse observe que dans la plupart des cas ceux-ci construisent leur récit en appliquant le principe d'ordre naturel, c'est-à-dire que

[...] les événements sont racontés dans l'ordre dans lequel ils ont eu lieu en réalité. La structuration du récit est fortement chronologique et linéaire. Pour ce qui est de la jonction propositionnelle, les apprenants se servent pour la plupart de ce que la tradition appelle la parataxe, c'.-à-d. des énoncés juxtaposés ou coordonnés, liés dans ce dernier cas par et ou mais (Buysse, 2016, pp. 2-3).

C'est seulement au fur et à mesure que leurs compétences linguistiques se développent que les élèves vont être capables de mobiliser des moyens linguistiques plus complexes.

Pendant un cours de langue, les participants peuvent être incités à créer soit un récit d'événements réels s'inspirant, par exemple, de leurs expériences personnelles, soit un récit de fiction. Ce deuxième prend souvent la forme d'une « activité suscitée [...] dont la source (support de l'activité) peut être verbale ou orale, ou comprendre même des données non-verbales (planches, bandes dessinées, film) » (PaprockaPiotrowska, 2003, p. 34). C'est justement ce deuxième type qui va nous intéresser dans le cadre de la présente étude, car nous allons nous focaliser sur les cas où les élèves réagissent à des séquences animées insérées dans les jeux vidéo.

\subsection{Raconter L'intro Du Jeu - Fragment of Dejavu}

Le premier jeu que nous allons décrire ici, intitulé Fragment of Dejavu, a été produit par un programmeur indépendant ReMind en utilisant la technologie flash. Le jeu s'ouvre avec une séquence composée d'une série de six images immobiles s'affichant successivement à l'écran sur lesquelles apparaît le héros principal accompagnant sa femme à l'aéroport, lui faisant des adieux et regardant son avion décoller de l'aéroport. L'avion explose - fait que nous apprendrons seulement dans la suite du jeu - à la suite d'un attentat terroriste. Ensuite, les six premières images disparaissent de l'écran et sont remplacées par une série d'images animées sur 
lesquelles est représentée la suite des aventures du protagoniste qui a entrepris le projet de construire une machine à voyager dans le temps. Son travail est accompli en 2021 lorsqu'il construit un engin qui lui permettra de retourner en 1999 et de tenter de déjouer l'attentat. Ce genre d'introduction (appelé souvent de manière abrégée - intro), présent dans de nombreux jeux vidéo, permet aux joueurs de comprendre le cadre dans lequel se déroulera l'intrigue du jeu.
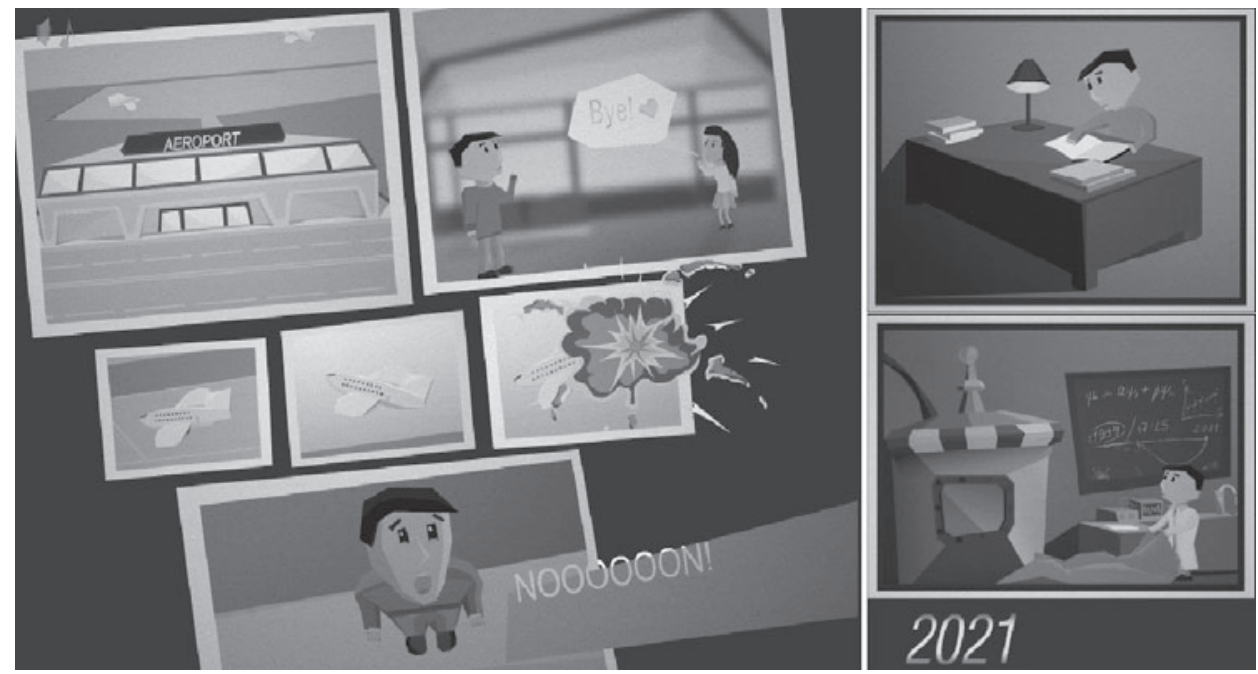

Illustration 1. L'intro du jeu Fragment of Dejavu. En premier lieu, à l'écran apparait la planche contenant les six premières illustrations (reproduite à gauche). Une fois que cette planche disparait, sont successivement affichées deux images animées reproduites à droite

Nous allons présenter ci-dessous deux fragments de leçons conduites dans deux classes différentes. La juxtaposition des transcriptions nous permettra d'observer deux réalisations potentielles de la tâche en question. Dans les deux cas, c'est l'enseignant qui joue le rôle d'initiateur, en posant la question «qu'est-ce qui s'est passé » et en encourageant ainsi les élèves à raconter l'histoire qu'ils ont vu se dérouler à l'écran.

\section{Fragment 1}

$01 \mathbf{N}$ : alors (.) qu'est-ce qui s'est passé (1.9)

02 U3: je:/je ne suis pas sûre mais un/un homme

(.) une femme a: eu un accident $\uparrow$ (.) d'avion $\uparrow$ (.)

$03 \mathbf{N}$ : oui absolument (.) quel type d'accident $\downarrow$ (.)

04 U3: il y avait une/une explosion et elle est morte

(.) et il a perdu la femme (.) 
U3:

\section{machine hmm (.) pour/pour changer:/changer} le temps $\uparrow$ (.)

13 U4: le passé (.) changer le passé(.)

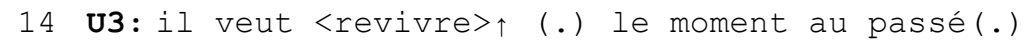

$15 \mathbf{N}$ : pourquoi $(0.8)$

16 U5: il veut retrouver sa femme et: faire la sauver(.)

$17 \mathbf{N}$ : il veut sauver la femme oui

Comme nous l'avons déjà dit, la séquence est initiée par la question de l'enseignant (01), qui s'adresse à l'ensemble des apprenants. Elle est suivie d'une réponse de $\mathrm{U} 3$, qui va poursuivre le récit pratiquement à sa conclusion malgré des interventions ponctuelles de ses collègues $(05,13,16)$. Quant à l'enseignant, son rôle principal est soit d'encourager le locuteur à poursuivre le discours et/ou le valider avec des adverbes affirmatifs (oui, absolument [03], oui [11, 17]), soit de relancer le récit en lui demandant directement de relater la suite des événements (07) ou d'expliquer les causes de tel ou tel événement (15). À part cela, l'enseignant tente de corriger certaines erreurs, p. ex. dans 09 il adresse à U3 une demande de clarification (clarification request) suite à l'emploi d'une forme (étant le résultat du transfert de la langue anglaise) que l'apprenant tente d'adapter à la prononciation française (stratégie appelée foreignizing par Dörnyei \& Scott, 1997, p. 189). À la suite de cette intervention, U3 est capable de s'autocorriger (10).

Quant à la construction du récit, il semble que les apprenants n'ont pas eu de difficultés à interpréter correctement le cours de l'histoire et en identifier les éléments constitutifs. Bien évidemment, lorsque le récit est présenté sous forme d'images mobiles dont le temps d'affichage est limité, il est possible qu'ils retiennent seulement un nombre limité d'informations. Il est incontestablement plus facile de garder une vision globale de l'ensemble lorsque toutes les illustrations sont simultanément accessibles au regard comme c'est le cas lorsque les élèves travaillent sur un support papier. Ici, les images disparaissent donc soit ils retiennent une image globale de l'histoire, soit ils mémorisent les éléments les plus saillants, mais pas nécessairement les plus importants du point de vue de la logique du récit, comme en témoigne l'intervention de U6 (05). U3 est capable de créer un récit cohérent, même si sa construction est plutôt simple, le discours étant marqué par l'utilisation temporelle du connecteur non-spécialisé et (Paprocka-Piotrowska, 2003, p. 105) qui 
sert à introduire les événements successifs (04, deux occurrences), stratégie utilisée aussi par ses collègues U6 et U5 $(05,16)$.

Les interventions de U4 et U5 qui apparaissent à la fin du passage cité (à partir de la ligne 13) sont éclairantes en ce qu'elles révèlent la manière dont les coparticipants peuvent aider le locuteur principal à compléter le discours narratif. Dans 13, U4 réagit spontanément à une construction qu'il juge incorrecte (changer le temps). Dans le tour de parole suivant, U3 reprend le récit en intégrant le mot passé dans son discours. Ensuite (16), c'est U5 qui prend la parole pour répondre à la question posée par l'enseignant relative aux motivations du héros principal du jeu. Le passage se clôt avec une reformulation de l'enseignant (17). Passons maintenant à l'analyse du fragment suivant, que nous reproduisons ci-dessous.

\section{Fragment 2}

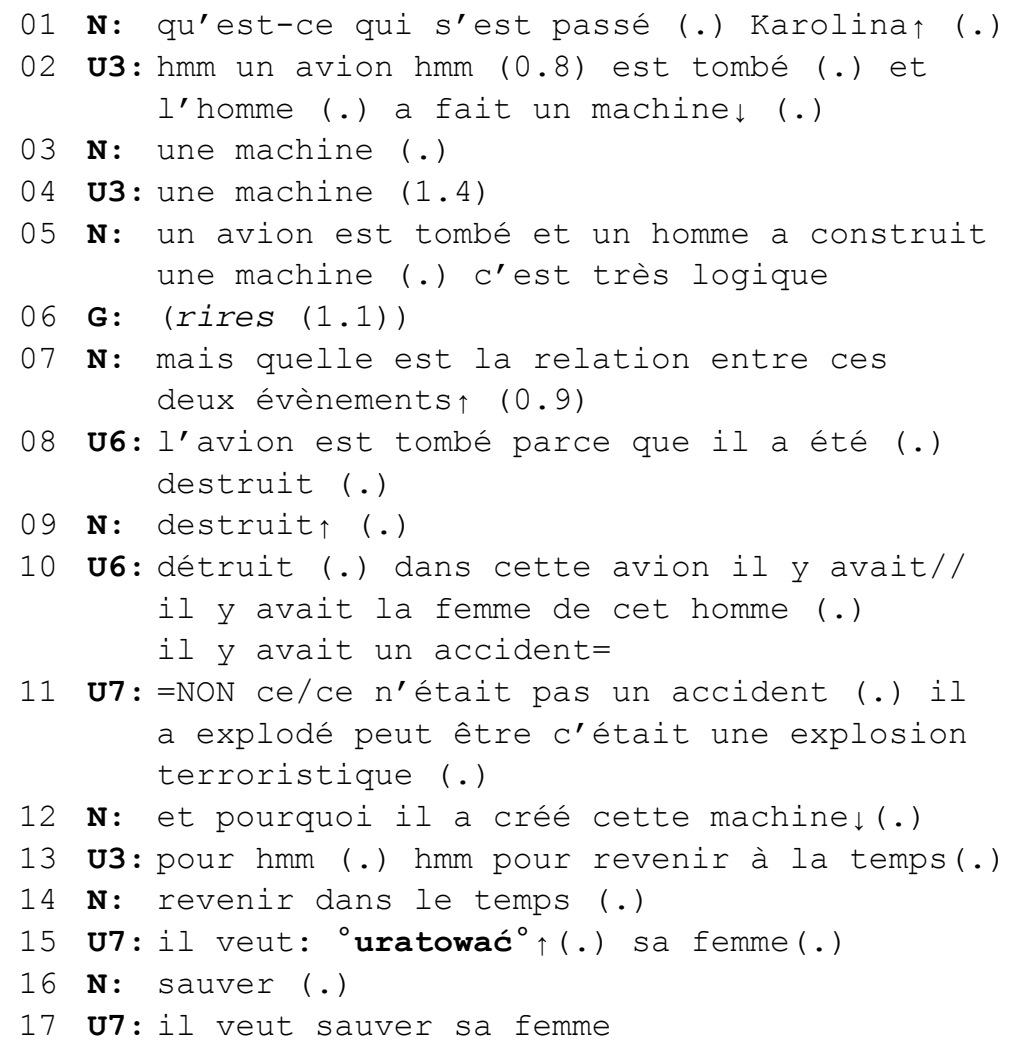

Cette fois-ci, le récit est construit d'une manière différente que précédemment. U3, sélectionnée par l'enseignant, peine à créer un récit cohérent, en ne produisant que deux phrases liées par la conjonction de coordination et (02). Non seulement 
cette production ne comporte aucun élément permettant d'identifier les éléments constitutifs du récit, ses acteurs ou son contexte, mais encore la relation entre les deux segments reste complètement obscure, fait sur lequel insiste l'enseignant dans 05 et 07 , la première de ces assertions se terminant par un éclat de rire des apprenants (06). Les difficultés linguistiques des apprenants étant nombreuses, les interventions de l'enseignant se font ici encore plus fréquentes : reformulations (ang. recasts ; 04, 14), répétitions de forme erronée (ang. repetitions, 09) et correction explicite de la forme employée par l'élève suite à une alternance codique de type insertionnel (ang. code-switching) employée par celui-ci (15). Il pose aussi à deux reprises $(07,12)$ des questions dont le but est d'encourager les apprenants à expliciter les relations de cause à effet entre les composantes du récit.

À partir de 08, deux autres apprenants (U6 et U7) vont prendre le relais et tenter de compléter l'histoire en y ajoutant les éléments nécessaires pour constituer un ensemble cohérent. Fait curieux, ils ne se satisfont pas de donner une vision objective des événements, mais tentent également d'émettre des hypothèses quant aux causes de l'accident. Ainsi, dans 11, U7 fait une irruption dans le discours de U6 (enchaînement immédiat, ang. latching) en opposant à son explication une autre, qui est d'après lui plus plausible. Il semble que le groupe s'accorde sur l'interprétation finale de l'histoire car cette interprétation n'est pas contestée par les autres apprenants. Le passage se termine encore une fois par une reformulation de l'enseignant.

\subsection{Placer l'intro du jeu dans un contexte plus large - Life is Strange}

Dans le jeu Life is Strange, édité en 2015 par le studio Square Enix, les joueurs incarnent Maxine Caulfield, étudiante en photographie qui commence à être hantée par d'étranges visions. Le jeu s'ouvre justement avec l'une d'entre elles, même si au début le joueur ne se rend pas compte qu'il s'agit d'une vision : Max se réveille dans la forêt pendant un violent orage, sans avoir le moindre souvenir des circonstances qui l'ont menée à cet endroit. Elle se dirige vers un phare qu'elle voit en haut de la colline et découvre à son horreur une immense tornade ravageant la ville. Un petit bateau de pêche est propulsé contre le phare et les débris tombants tuent Max ; c'est alors qu'elle se réveille, désorientée, en classe.

Dans le fragment de la leçon de français que nous citons ci-dessous, la narration est déclenchée par un événement ayant eu lieu plus loin dans le jeu: les élèves aperçoivent une photo punaisée sur le tableau en liège dans la salle de classe de Max et reconnaissent le phare qui était présent dans sa vision (01-02). U6, qui contrôle les mouvements du personnage, ne comprend pas les propos de ses collègues en raison de son absence pendant le dernier cours et le signale à l'enseignant (03). C'est la raison pour laquelle celui-ci prie U5 de récapituler ces événements (06). 


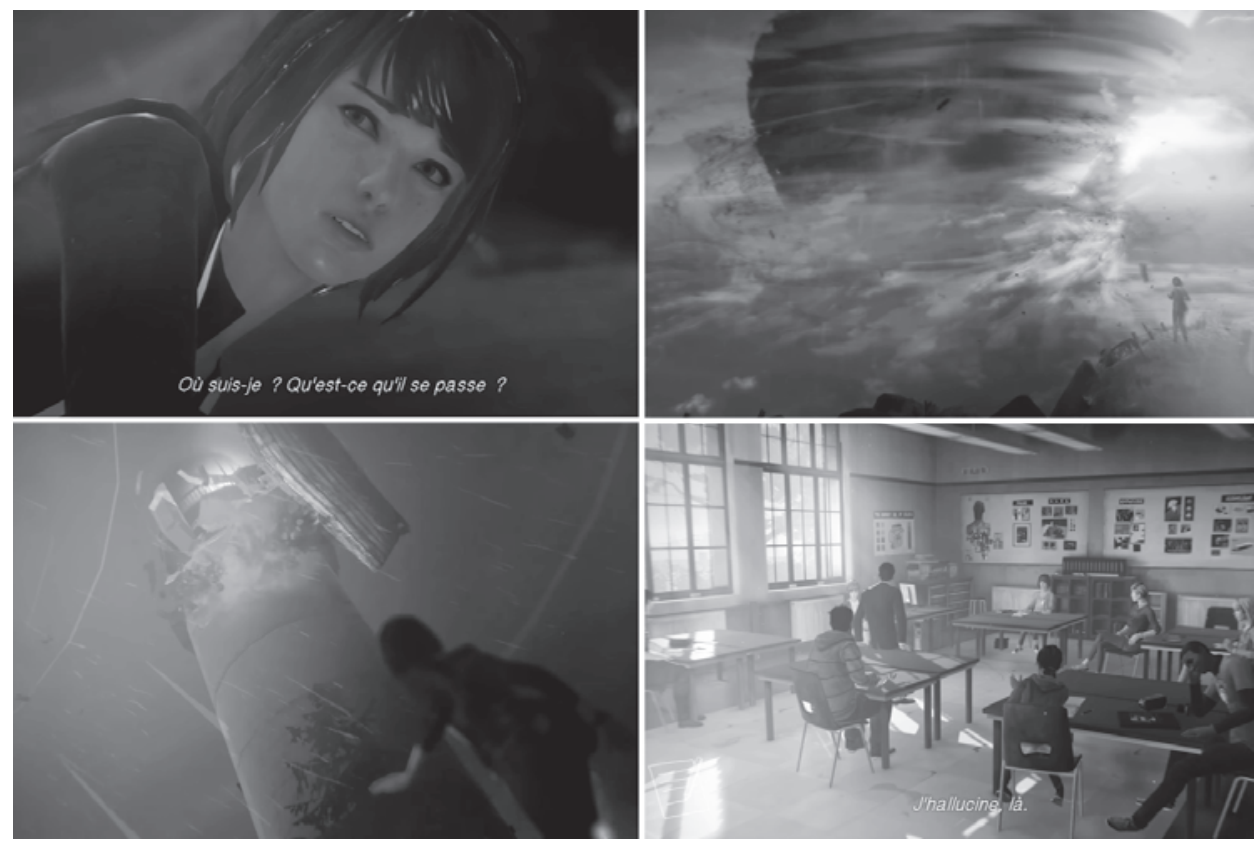

Illustration 2. Quelques cadres choisis de l'intro du jeu Life is Strange

\section{Fragment 3}

01 U4: là (.) regarde sur la photo là (.) c'est le phare qqui ...?...

$02 \mathrm{U1}$ : \{OUI C'est le même donc c'est/ c'est réel (1.1)

03 U6: alors ce phare (.) c'est quoi (.)

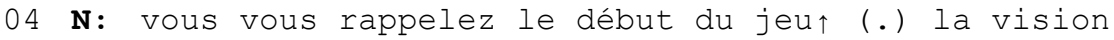

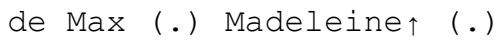

05 U6: j'étais absente (.)

$06 \mathbf{N}$ : Sophie, tu peux nous rappeler ce qui s'est passé avant $\uparrow(1.6)$

07 U5: hmm alors Max avait des: (.) elle était dans la classe et elle avait le rêve//la rêve quelque chose comme ça qu'elle était dans la forêt et elle est entrée en tempête mais elle a dit que/que ce n'était pas un rêve et que c'était très hmm très réel (.)

08 U2: c'était une hallucination (.)

09 U5: oui et puis des hmm des choses hmm elle a il y avait des / des pièces ...?... qui/qui (.) qui sont tombées sur / sur elle et quand elle (.) et quand elle était 


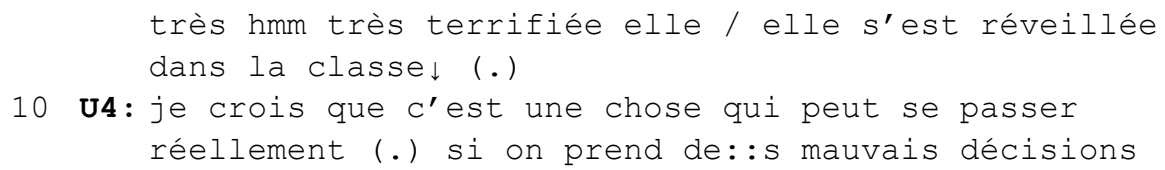

U5 tente de créer un récit cohérent, tout d'abord en essayant de l'encapsuler dans un cadre qui rendra possible son insertion ultérieure dans un contexte plus large. Même si dans le jeu l'histoire s'ouvre à l'instant où Max gît, inconsciente, dans la forêt, et c'est seulement à la fin de la séquence animée que l'on découvre qu'il s'agissait d'une vision/rêve du protagoniste, l'élève choisit d'expliciter d'emblée le statut de l'histoire en en marquant le début et l'achèvement (elle était dans la classe et elle avait un rêve $\neq$ elle s'est réveillée dans la classe). La construction du récit progresse d'une manière plutôt fluide malgré les pauses et répétitions fréquentes, ainsi que l'emploi prononcé de l'onomatopée $\mathrm{hmm}$ marquant son hésitation. Mis à part la conjonction et servant à introduire les étapes successives du récit, U5 est également capable de mobiliser certains connecteurs temporels (quand, puis ; 09) et logiques (mais, 07).

Contrairement aux deux cas que nous avons présentés dans la section 3.1, ici, l'élève est capable de construire un récit d'une manière entièrement autonome. U5 ne recourt ni à l'aide de l'enseignant ni à celui de ses collègues. Le produit final est cohérent et permet à l'auditeur d'identifier correctement les étapes successives de l'histoire. Les deux interventions de U2 et U4 qui apparaissent au milieu (08) et à la fin du récit (10) ne servent pas à la compléter, mais jouent le rôle de commentaire dont la fonction est de placer les événements relatés dans un contexte plus large. Surtout la deuxième remarque, celle de U4, est ici révélatrice, car elle permet à l'ensemble des participants de comprendre la véritable signification de la scène relatée par U5. L'intro n'est pas une banale séquence animée destinée à attiser l'attention du spectateur ou - dans le contexte d'une classe de langue - un pur prétexte pour stimuler l'activité langagière des apprenants. Elle est une composante essentielle de l'expérience ludique qu'il est nécessaire de placer dans le contexte approprié afin d'être capable de mener le jeu vers le dénouement désiré. La séquence relatée par U5 est ainsi identifiée - tout à fait correctement - comme la projection d'une fin qu'il va falloir éviter.

\subsection{Jeu 3 - Bermuda Escape - rappel des actions passées}

Le jeu suivant, Bermuda Escape, a été produit en 2009 par le studio Pastel Games. Cette fois-ci les joueurs se retrouvent en plein milieu du triangle des Bermudes sur un bateau abandonné. Les tentatives d'allumer le moteur ou d'appeler à l'aide s'avèrent infructueuses. Alors que le joueur explore le cockpit, une énorme vague coule le bateau, et à partir de ce moment l'objectif sera de trouver une façon de se 
libérer de l'épave. Cette fois-ci, l'élève (U3) ne relate pas l'introduction du jeu mais rend compte des actions qui ont été menées lors du cours précédent. Le jeu réalisé en technologie flash ne permettant pas d'effectuer la sauvegarde, il est nécessaire de revenir brièvement sur les événements qui ont eu lieu pendant la dernière séance.

\section{Fragment 4}

$01 \mathbf{N}$ : oui alors qu'est-ce qui s'est passé

la dernière fois (1.9)

02 U3: alors hmm nous avons pris: hmm [selobje]

(montre un des objets avec le doigt) alors

(prend I'objet) ce objet rouge (.) la masque (.)

$03 \mathbf{N}$ : le masque (.)

04 U3: le masque et/et hmm [a:] (0.7) nous voyons hmm

(.) qu'il y a <une mappe> (.)

$05 \mathbf{N}$ : une quoi $\uparrow(0.7)$

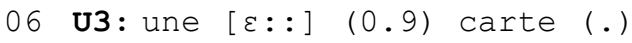

$07 \mathbf{N}$ : carte (.)

08 U3: carte (.) oui (appuie sur deux boutons)

alors hmm nous pouvons voir hmm (1.1)

(montre avec le doigt) où nous se:/se trouve (.)

$09 \mathbf{N}$ : où on se trou $\cdots$ ve oui

10 U3: $\quad$ où on se trouve hmm et

[Es] la clé ne marche pas (touche la clé)

et (change la position des leviers) les leviers

aussi hmm (1.4)

$11 \mathbf{N}$ : quoi encore $\uparrow(0.7)$

12 U3: [E:] il y avait: des objets dans ce/ce (.) tiroir (ouvre le tiroir à droite) ah oui (prend deux objets) c'est [k.: : ] (0.9) >COMBINAISON< (.)

et ici (ouvre le tiroir à gauche) un livre (.) c'est le journal de bord (tout à coup le bateau coule reversé par une grande vague (3.7))

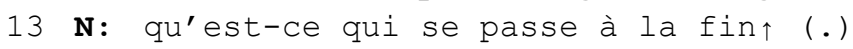

14 U5: le bateau COULE

Le fragment présenté ci-dessus présente un autre cas d'interaction élèveprofesseur. Une fois de plus, le rôle de celui-ci se borne à poser des questions encourageant l'apprenant à relater les étapes successives de l'action (01, 11 i 13) ainsi qu'à fournir le feedback correctif, en utilisant les reformulations d'une forme erronée (ang. recast ; 03, 09) ou des demandes de clarification (ang. clarification request, 05). Quant à l'activité de l'apprenant, celle-ci se décline sur deux plans différents : d'une part, U3 relate les événements ayant eu lieu pendant la séance précédente, d'autre part elle reproduit les actions d'un autre participant. 
U3 utilise la première personne du pluriel (nous, 02, 04, 08), ce qui prouve qu'elle se perçoit elle-même, ainsi que ses collègues, comme membres d'une communauté de joueurs. La verbalisation des étapes successives est synchronisée avec l'exécution des actions qui leur correspondent. Cette activité se déroule simultanément sur deux plans temporels : présent et passé. En témoigne l'utilisation alternative de l'indicatif présent $(04,08$ et 10$)$, du passé composé $(02)$ et de l'imparfait (12). Cette oscillation visible chez U3 est facilement compréhensible, car chacun des énoncés peut être vu sous deux angles différents : celui d'une pure description de l'état d'un objet donné (p.ex. la clé ne marche pas, 10) ou bien celui de l'évocation - et réplication synchronisée - des activités effectuées par un autre élève lors du cours précédent (p.ex. nous avons pris cet objet, 02).

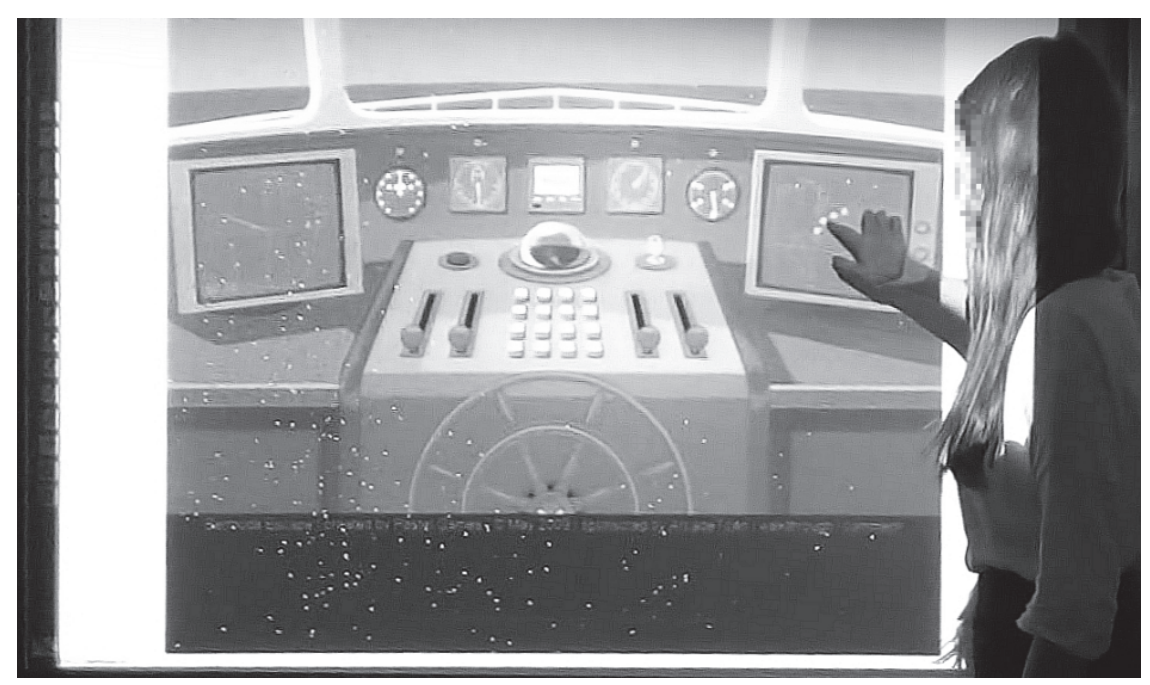

Illustration 3. U3 montre du doigt la carte sur laquelle est visible le trajet parcouru par le bateau (08)

Les activités de ce genre permettent également à l'apprenant d'utiliser dans un contexte précis le vocabulaire acquis précédemment. Ce rappel du vocabulaire ne se déroule pas toujours sans difficultés : alors que certains items (p. ex. leviers, 10) sont aisément intégrés dans le discours, d'autres (p.ex. tiroir et combinaison, 12), exigent chez U3 un effort de remémoration plus important, comme en témoignent les répétitions et le changement du débit de la parole de l'élève. D'autre part, certains items sont restés particulièrement bien gravés dans la mémoire des participants, comme en témoigne l'intervention spontanée de U5, qui rompt la communication dyadique entre l'enseignant et U3 en évoquant le verbe couler avec un accent d'insistance marqué par le volume de la parole élevé. 


\subsection{Jeu 4 - Fog Fall 3 - raconter une intrigue secondaire}

Le dernier exemple que nous allons analyser dans le cadre de la présente étude est celui d'une intrigue secondaire (ang. subplot), qui n'est pas un élément essentiel de l'histoire, mais peut également être un bon prétexte pour encourager les participants à produire un récit dont l'objectif est la reconstruction des événements qui se sont déroulés à un certain moment du jeu.
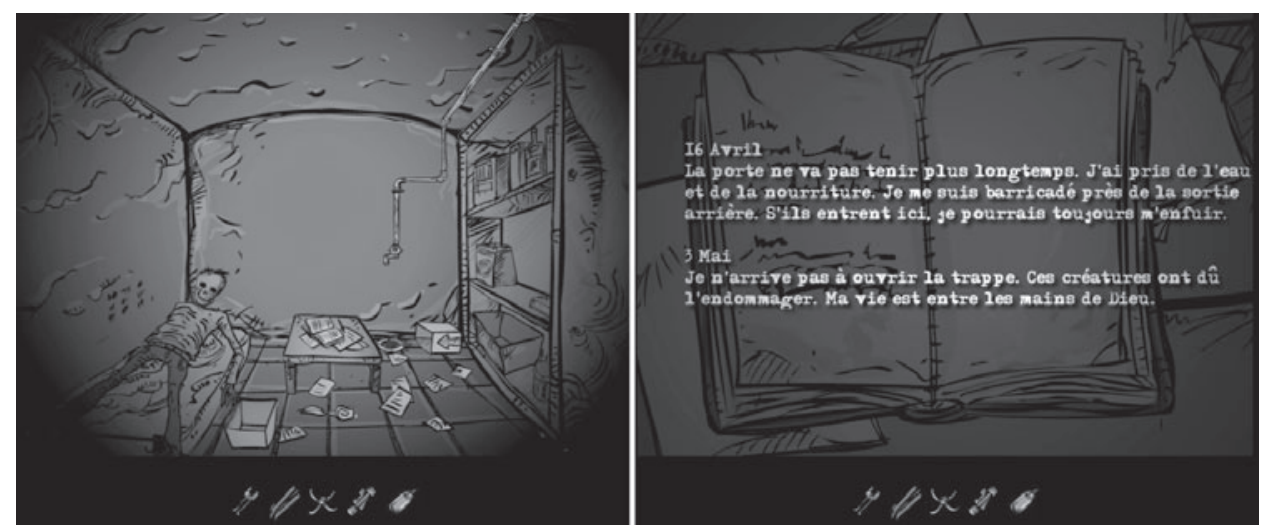

Illustration 4. Deux cadres du jeu Fog Fall 3. À gauche la vue d'une des chambres du refuge, à droite le journal intime de l'homme retrouvé mort.

Le logiciel qui va nous servir d'exemple, Fog Fall 3, a été créé en 2010 par le studio polonais Pastel Games. Dans ce jeu, les joueurs évoluent dans un univers post-apocalyptique dans lequel les hommes combattent pour trouver des ressources indispensables à leur survie. À un moment, les participants trouvent un passage bloqué, qui, une fois dégagé, mène à un abri antiatomique. Dans une des pièces, ils retrouvent un homme mort et sont encouragés par l'enseignant à spéculer sur la suite d'événements qui a mené à cette tragédie.

\section{Fragment 5}

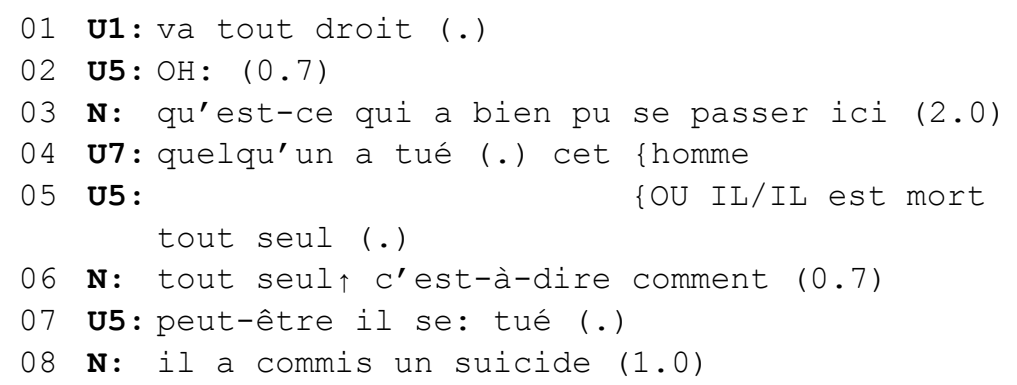


09

U2: ou quelqu'un est venu et/et il voulait: prendre

(.) ses choses (1.4)

10 U1: tu/tu regardes la table (.) là (elle pointe avec le doigt)

11

12

U2: (clique sur l'objet) c'est un journal (.)

N: tu peux lire Anna $\uparrow(0.8)$

$\mathrm{U}$ : (lit le texte (31.8))

$\mathbf{N}: \operatorname{alors} \uparrow(1.6)$

U3: alors cet homme (.) il habitait peut-être dans cette/cette maison (.) et: un jour quelqu'un a/a attaqué (.) cet homme et il est allé ici pour se:/se cacher (.) et ensuite quelqu'un voulait entrer là et: il s'a bloqué $\uparrow$ (.) // il a bloqué la porte et il ne pouvait pas sortir et/et quand il n'avait rien pour manger il est mort de/de: (.)

16 U2: $\operatorname{faim}_{\uparrow}($.

$17 \mathbf{N}$ : à la fin il est mort de faim oui

La construction du récit se déroule ici en deux temps : d'abord, les élèves, en se basant sur quelques indices visuels, essaient de déterminer la cause la plus probable du décès de l'homme retrouvé dans le refuge (04-09). Les raisons proposées par U7 et U5 (c'est-à-dire que sa mort résulte d'un meurtre ou d'un suicide) sont les plus probables. U2 tente de développer sa réponse vis-à-vis de l'idée formulée par U7 en essayant de proposer une explication plausible sur la raison pour laquelle un tiers aurait commis le crime en question (09). En effet, la volonté de voler les provisions de l'homme se cachant dans l'abri est un mobile tout à fait probable vu que le jeu se déroule dans un monde post-apocalyptique dans lequel le combat pour les ressources alimentaires se déroule au quotidien. C'est en découvrant le journal intime du personnage que les apprenants sont capables de reconstruire la suite des événements qui ont conduit à sa mort, tâche réalisée par U3 (15).

U3 est capable de construire le récit d'une manière autonome; seulement à son achèvement se manifeste l'insuffisance de ses moyens lexicaux. L'intervention spontanée de U2 permet pourtant de compléter cette lacune (16). La structure du récit est simple et linéaire: une fois de plus, les événements successifs sont introduits à l'aide du connecteur non-spécialisé et, une seule occurrence de connecteur spécialisé (ensuite) étant relevée. Cependant, l'apprenant semble avoir bien maîtrisé l'emploi du passé composé et de l'imparfait, correctement employés pour décrire l'état et les événements ponctuels.

Cette fois-ci, la création du récit n'est pas strictement parlant nécessaire pour comprendre les événements essentiels pour le jeu. Pourtant, elle permet de mieux comprendre la réalité dans laquelle se déroulent les événements, ainsi que d'entraîner l'implication émotionnelle des apprenants en renforçant le sentiment d'immersion. 


\section{CONCLUSION}

Dans le cadre de notre étude, nous avons été contraints de nous limiter à présenter seulement quelques cas où les activités vidéoludiques, intégrées dans la pratique de classe de langue, permettent de créer un contexte propice à ce que la narration se manifeste dans le discours des apprenants. Faute de place, il nous a été impossible de mener une analyse approfondie du phénomène. Pourtant, même cette étude de dimensions restreintes montre clairement que les stratégies vidéoludiques peuvent stimuler l'activité langagière des apprenants du FLE, et qu'il s'agit d'un terrain de recherche prometteur.

L'un des domaines qui mériterait certainement une investigation approfondie dans le cadre d'une recherche ultérieure est le rapport entre le type de support utilisé et la qualité du récit produit par les apprenants. Ainsi, il serait intéressant de comparer la production orale des élèves prenant part aux activités vidéoludiques avec celle des apprenants décrivant une suite d'images lors d'un exercice narratif plus « classique ». Certains récits présentés sous forme graphique, telle que, par exemple, une bande dessinée, peuvent s'avérer particulièrement difficiles à reconstruire sans un contexte approprié ; c'est précisément dans ce genre de cas que les avantages des jeux vidéo pourraient se manifester.

Une autre question est celle de l'implication émotionnelle dans une histoire à laquelle les élèves participent et qu'ils « vivent » lorsqu'ils jouent à un jeu vidéo. La reconstruction d'une histoire à partir d'un support papier peut être perçue comme un pur exercice linguistique sans relation quelconque avec les autres activités de classe. Dans le cas des pratiques vidéoludiques, la construction du récit se trouve naturellement intégrée dans le processus du jeu. La plupart des jeux racontent une histoire et placent l'utilisateur dans un univers fascinant que le joueur se sent motivé à découvrir. Cette réalité virtuelle complexe, composée d'une suite de récits enchevêtrés, peut sans aucun doute constituer un cadre propice à l'expression langagière de l'apprenant. Bien évidemment, cela ne veut pas nécessairement dire que, par exemple, l'envie de communiquer des apprenants, appelée en anglais willingness to communicate, sera à chaque fois supérieure dans le cas des activités narratives vidéoludiques par rapport aux activités traditionnelles. Le fait de suspendre momentanément le jeu peut créer un effet tout à fait inverse à nos attentes. Les élèves, désirant reprendre la partie qui a été arrêtée, peuvent considérer l'exercice de narration imposé par l'enseignant comme une intervention agaçante interrompant l'activité en cours.

Finalement, il faut également prendre en considération une autre composante essentielle de l'équation : la volonté et les compétences de l'enseignant. L'incorporation des techniques numériques dans une classe de langue nécessite un effort considérable de la part du professeur, en ce qui concerne le choix du matériel et du 
logiciel, l'assignation des rôles aux acteurs de l'interaction, le contrôle du flux d'information dans la classe, etc. Cela nécessite une préparation méticuleuse préalable, la maîtrise des outils informatiques et, avant tout, la volonté de sortir des sentiers battus. Dans notre article, nous avons tenté de montrer que cet effort peut souvent s'avérer fructueux. 


\section{BIBLIOGRAPHIE}

Buysse, M. (2016). Dépendance sans intégration. La cosubordination comme mode de jonction propositionnelle et sa pertinence en acquisition du français L2. In F. Neveu, G. Bergounioux, M.-H. Côté, J.-M. Fournier, L. Hriba, \& S. Prévost (Eds.), Congrès Mondial de Linguistique Française - CMLF 2016 (SHS Web of Conferences ; vol. 27). EDP Sciences-Web of conferences. DOI : $10.1051 /$ shsconf $/ 20162710003$.

Dörnyei, Z., \& Scott, M.L. (1997). Communication Strategies in a Second Language. Definitions and Taxonomies. Language Learning, 47 (1), 173-210. DOI : 10.1111/0023-8333.51997005.

Gee, J.P. (2008). What video games have to teach us about learning and literacy. Basingstoke : Palgrave Macmillan.

Johnson, S. (2005). Everything bad is good for you. New York : Riverhead Books.

Kotuła, K. (2015). New technologies and classroom interaction. Computer-enhanced ludic techniques in language learning. In A. Turula, B. Mikołajewska, \& D. Stanulewicz (Eds.), Insights into Technology Enhanced Language Pedagogy (pp. 79-90). Frankfurt am Main : Peter Lang. DOI : $10.3726 / 978-3-653-04995-4$.

Kotuła, K. (2016). Le rôle de l'enseignant d'une langue étrangère dans un environnement numérique. Des mondes virtuels aux jeux vidéo. Romanica Cracoviensia, 16 (2), 97-107. DOI : 10.4467/20843917RC.16.009.5930.

Mawer, K., \& Stanley, G. (2011). Digital play: computer games and language aims. Surrey : Delta Publishing.

Paprocka-Piotrowska, U. (2003). Temporalité et narration. Développement des moyens de donner l'information temporelle dans le discours narratif chez des apprenants polonophones débutant français. Lublin : Towarzystwo Naukowe Katolickiego Uniwersytetu Lubelskiego.

Paprocka-Piotrowska, U. (2013). Construction du récit dans la communication en langue étrangère. Lublin : Wydawnictwo Werset.

Prensky, M. (2006). Don't Bother Me Mom - I'm Learning! Saint Paul, Minnesota : Paragon House.

Reinders, H. (Ed.) (2012). Digital Games in Language Learning and Teaching. Palgrave Macmillan. DOI : $10.1057 / 9781137005267$.

Reinhardt, J., \& Sykes, J.M. (Eds.) (2014). Game-informed L2 Teaching and Learning [Special issue]. Language Learning \& Technology, 18 (2). Retrieved from http://www.lltjournal.org/ collection/col_10125_35926.

Tettegah, S., McCreery, M., \& Blumberg, F. (Eds.) (2015). Psychological Perspectives on Digital Games and Learning [Special issue]. Educational Psychologist, 50 (4). Retrieved from https://www.tandfonline.com/toc/hedp20/50/4?nav=tocList.

Thomas, M. (2012). Contextualizing Digital Game-Based Language Learning. Transformational Paradigm Shift or Business as Usual? In H. Reinders (Ed.), Digital Games in Language Learning and Teaching (pp. 11-31). New York : Palgrave Macmillan. DOI : 10.1057/9781 137005267_2. 